\title{
Boundary conditions and vortex wandering
}

\author{
S. P. JAMMY, NICK HILLS AND DAVID M. BIRCH \\ Department of Mechanical Engineering Sciences, \\ University of Surrey, Guildford, Surrey GU2 7XH UK
}

(Received 27 November 2013)

\begin{abstract}
A direct numerical simulation of a Batchelor vortex has been carried out in the presence of freely-decaying turbulence, using both periodic and symmetric boundary conditions; the latter most closely approximates typical experimental conditions, while the former is often used in computational simulations for the purposes of numerical convenience. The higher-order velocity statistics were shown to be strongly dependent upon the boundary conditions, but the dependence could be mostly eliminated by correcting for the random, Gaussian modulation of the vortex trajectory commonly referred to as 'wandering' using a technique often employed in the analysis of experimental data. Once corrected for this wandering, the strong peaks in the Reynolds stresses normally observed at the vortex centre were replaced by smaller local extrema located within the core region but away from the centre. The distributions of the corrected Reynolds stresses suggested that the formation and organization of secondary structures within the core is the main mechanism in turbulent production during the linear growth phase of vortex development.
\end{abstract}

Key Words: turbulent flows, vortex dynamics, vortex flows

\section{Introduction}

Vortex flows remain a subject of considerable interest, not only because of their fundamental importance, but because of their value in a wide variety of applications. At the smallest scales, vortices can be considered as the basic elements of turbulence and are directly responsible for dissipation; at larger scales, they may be controlled to enhance mixing or the lift generated by flapping wings (Froehlich et al. 2008; Wang 2005). At even larger scales, they are entirely responsible for the induced drag generated by aircraft (Spalart 1998; Kroo 2001); at atmospheric and planetary scales, they are of interest in meteorological modelling and storm prediction (Kurihara et al. 1993; Zhang \& Sarkar 2012).

In experimental studies of vortex flows, a quasi-two-dimensional or trailing vortex is typically produced using a vortex generator composed of one or more lifting bodies, installed in a flow facility. Once allowed sufficient streamwise distance to achieve axisymmetry, the resultant vortex begins to closely approximate the Batchelor (1964) profile, so that

$$
\frac{V_{\theta}(\eta)}{V_{0}}=\left(1+\frac{1}{2 \alpha}\right) \frac{1}{\eta}\left[1-\exp \left(-\alpha \eta^{2}\right)\right],
$$

where $V_{\theta}(\eta)$ is the tangential velocity, $V_{0}=V_{\theta}(1)$ is the maximum tangential velocity, $\eta=r / r_{c}$ is the radial coordinate, $r_{c}$ is the core radius (or the radial location at which $V_{\theta}=V_{0}$ ), and $\alpha \sim 1.256$ is Lamb's constant (uppercase variables here are used to indicate time-mean or time-invariant quantities). Batchelor's solution also admits a Gaussian 
distribution of axial velocity,

$$
\frac{V_{z}(\eta)}{W_{0}}=\exp \left(-\alpha l^{2} \eta^{2}\right)
$$

where $W_{0}$ is the peak axial velocity (which may be positive or negative), and $l$ is a rescaling parameter for the axial profile. Good agreement is achieved with (1.1) and (1.2) over a wide range of Reynolds numbers and boundary conditions (see Birch et al. 2004), including cases of highly unsteady vortices (Ramaprian \& Zheng 1998; Birch \& Lee 2005) and those subjected to intense free-stream turbulence (Beninati \& Marshall 2005; Bailey \& Tavoularis 2008).

However, all vortices generated within experimental flow facilities are acknowledged to be subject to a low-amplitude, random 'wandering'; this phenomenon results in a Gaussian distribution of the locus of vortex centres in both time and space. The process of vortex wandering and its significant impact on experimental measurements was reviewed in detail by Devenport et al. (1996). Though its mechanisms are still not understood, vortex wandering is believed to be a consequence of the initial and boundary conditions inherently imposed by wind tunnels, owing to the conspicuous absence of this phenomenon in free-flight tests (McCormick et al. 1968). The wandering amplitude is also highly sensitive to free-stream turbulence intensity, which likewise vanishes in an ideal free-flight test (van Jaarsveld et al. 2011; Bailey \& Tavoularis 2008).

The experimental characterization of vortex flows, however, remains limited by current measurement technology. Conventional planar and tomographic particle-image velocimetry (PIV) systems can resolve the instantaneous vortex structures, but tend to have fairly low bandwidths (relative to the inertial-range timescales). Although high-speed PIV capability is advancing rapidly, high-Reynolds number vortices are difficult to seed effectively (Birch \& Martin 2013). On the other hand, pointwise measurements, such as those from multi-component thermal anemometery probes or laser-Doppler velocimeters, can have a very high bandwidth but are fundamentally incapable of resolving structures. Most experiments are therefore limited in that the vortex may be well-resolved either in space or in time, but not both.

High-confidence, time-resolved turbulence data is therefore usually obtained using local measurements, and the contamination of the velocity statistics by the random, passive wandering of the vortex must be either corrected or removed. Isolating the effect of the vortex wandering and correcting the velocity statistics accordingly can be difficult, as this usually requires some a priori assumptions about the velocity distributions (Devenport et al. 1996; Iungo et al. 2009); furthermore, a number of sources of experimental error have been shown to artificially enhance the agreement between pointwise measurements and (1.1) (Birch 2012). Alternatively, multiple simultaneous local measurements within a vortex field will allow the re-mapping of the vortex to remove the effect of wandering. This generalized approach is more intrusive, and will also require some assumptions about the form of the vortex (Bailey \& Tavoularis 2008).

Direct numerical simulation (DNS) is therefore a very valuable tool in understanding the structure and dynamics of vortices, as it is entirely free from experimental error, and can provide results which are simultaneously well-resolved in space and time. However, computational simulations require that the governing equations be solved within some finite domain; this necessarily imposes the requirement of boundary conditions. Since boundary conditions are already understood to play a fundamental role in vortex wandering, it is reasonable to expect vortices modelled via DNS to likewise wander within the computational domain. Indeed, behaviour consistent with bulk wandering may be 
observed in some of the available published data (Melander \& Hussain 1993; Pradeep \& Hussain 2004).

In DNS simulations of nominally unbounded vortex flows, the boundary conditions are typically selected as a matter of computational convenience. However, because the vortex wandering is boundary condition-dependent and because the velocity statistics can be significantly affected by the wandering, the statistics themselves are also boundary condition-dependent. Since vortex wandering is an 'inactive' phenomenon (causing bulk advection but negligible net global production or dissipation), it will necessarily mask any similarity characteristics in much the same way as has been previously observed in the case of turbulent boundary layers (Townsend 1961; Bradshaw 1967).

To address this problem, one possible approach would be to significantly increase the size of the domain. However, because the mechanisms behind wandering remain unclear, no causal relationship between wandering amplitude and domain size is known to exist. In wind tunnel experiments, vortex wandering is universally observed - even in cases where the integral scale of the flow facility is orders of magnitude larger than the length scale of the vortex (see, for example Iungo et al. 2009). By comparison, DNS studies of vortex dynamics have domains typically limited to less than $\sim 30 r_{c}$ by the computational resources available (Pradeep \& Hussain 2004); simulating a sufficiently large domain to altogether eliminate vortex wandering may therefore be intractable with current technology. On the other hand, another possible approach would be to use a smaller domain but remove the effects of vortex wandering from the results a posteriori. This would provide an insight into the generalized dynamics of vortex development, independent of the specific (and arguably arbitrary) boundary conditions used.

The purpose of this paper, then, is to $(a)$ demonstrate by example the influence that specific boundary conditions may have upon velocity statistics in vortex flow simulations as a consequence of vortex wandering; $(b)$ demonstrate that a correction for vortex wandering can yield velocity statistics which are boundary condition-independent and therefore more likely to be representative of unbounded vortices, and, by so doing, $(c)$ facilitate the characterization of the structure of these complex flow structures.

\section{Numerical method}

In order to address the points raised above, direct numerical simulations of a Batchelor vortex were carried out in the presence of background turbulence. This formulation has been used extensively for fundamental numerical studies of turbulent vortex flows; for additional details, the reader is referred to Pradeep \& Hussain (2004), Goto (2008) and Duraisamy \& Lele (2008).

\subsection{Vortex model}

The incompressible continuity and Navier Stokes equations,

$$
\begin{aligned}
\nabla \cdot \mathbf{U} & =0 \\
\frac{\partial \mathbf{U}}{\partial t} & =-\frac{1}{\rho} \nabla p-\frac{1}{2}[\nabla(\mathbf{U} \otimes \mathbf{U})+(\mathbf{U} \cdot \nabla) \mathbf{U}]+\nu \nabla^{2} \mathbf{U}
\end{aligned}
$$

(where $\mathbf{U}$ is the velocity vector, $p$ is the pressure, $\rho$ is the density, $t$ is the time and $\nu$ is the kinematic viscosity) were solved on a regular, structured, $129 \times 129 \times 129$ point grid extending $20 \times 20 \times 40$ in $x / r_{c}, y / r_{c}$ and $z / r_{c}$, respectively (where $z$ is the axial direction), resulting in an isotropic grid spacing equivalent to twice the Kolmogorov length scale. The equations were solved in the spatial domain, in order to facilitate the implementation of arbitrary boundary conditions. A sixth-order compact difference scheme was used, with 


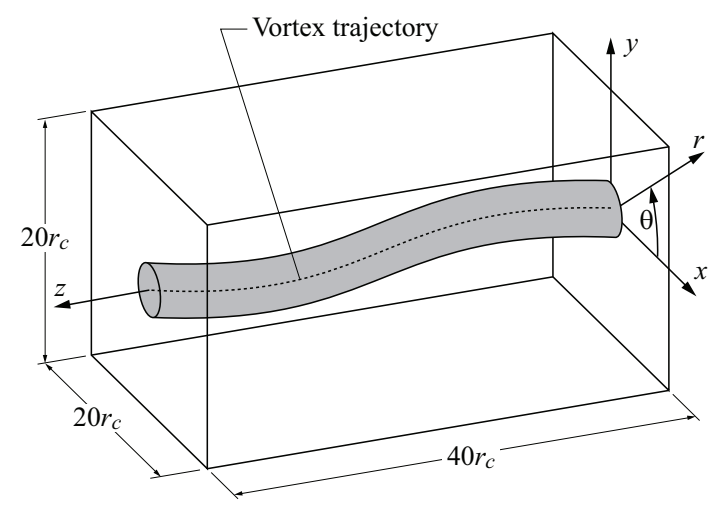

FiguRE 1. Illustration of the computational domain.

third-order, low-storage Runge-Kutta time-stepping. Convective and viscous fluxes were resolved using the technique of Lele (1992), with sixth-order accuracy. Continuity was enforced by solving the Poisson equation for pressure using a direct solver in spectral space (see Laizet \& Lamballais 2009, for details). Figure 1 shows a schematic illustration of the computational domain, including the coordinate systems used.

The boundary conditions were periodic in $z$, and solutions were obtained using both symmetric and periodic boundary conditions in $x$ and $y$ in order to provide a comparative measure of boundary condition sensitivity. It is important to note that although periodic boundary conditions are naturally implemented when solving the governing equations in the spectral domain, symmetric boundary conditions (which impose vanishing wall-normal velocities at the boundaries) are a more representative model of typical experimental conditions.

The solution was initialized using the two-dimensional Batchelor vortex mean velocity field described by (1.1) and (1.2). The tangential and axial velocity magnitudes were constrained by imposing a swirl parameter $q=1$, where

$$
q=\left(\frac{2 \alpha+1}{2 \sqrt{\alpha}}\right) \frac{V_{0}}{W_{0}}
$$

and an axial scaling parameter $l=1$. The core Reynolds number $R e_{c}=\Gamma_{c} / \nu$ was initially set to $4 \times 10^{3}$ (where $\Gamma_{c}$ is the circulation about the circular path given by $\eta=1$ ).

\subsection{Background turbulence}

In order to model free-stream turbulence (and provide a perturbation for the vortex), a synthetic field of homogeneous, isotropic turbulent velocity fluctuations was superimposed upon the mean velocity field in the initial condition. The fluctuations had a prescribed Gaussian energy spectrum $E(\kappa)$ (where $\kappa$ is the wavenumber), such that

$$
E(\kappa) \propto \kappa^{2} \exp \left(-\kappa^{2}\right)
$$

and were constrained to satisfy continuity. The synthetic turbulence was generated by assigning random phases to the velocity field in Fourier space, and mapping the resultant velocity field back into the physical domain by means of a three-dimensional inverse Fourier transform. This technique is described in detail by Qin (1998).

\subsection{Post-processing}

The velocity fields obtained with the DNS model were corrected for vortex wandering in post-processing. At each time step, the vortex centre (taken as the location of minimum 
tangential velocity) was identified on individual cross-flow $(x-y)$ planes using a thirdorder sub-grid interpolation. The vortex trajectory $x_{c}(z), y_{c}(z)$ was then identified as a the locus of these points of minimum tangential velocity. To remove the influence of wandering, the velocity data on each cross-flow plane were simply subjected to a planar translation, so that $x_{c}(z)$ and $y_{c}(z)$ would both be forced to vanish. In so doing, the axis of the vortex was forced to lie on the $z$ axis, thereby mapping each instantaneous vortex trajectory onto the trajectory of the initial condition. Because the curvature of the trajectory was negligible, no out-of-plane rotation or scaling of the cross-flow velocity fields was required.

It should be noted here that, both for the implementation of the wandering correction and subsequent conversion to polar coordinates, the definition of the vortex centre is critical and can have a significant effect upon the results, especially if the vortex becomes distorted. For a discussion of the different techniques of vortex identification and the advantages associated with each, the reader is referred to Giuni (2013). The criterion used here to identify the vortex centre was selected specifically for its robustness in ensuring that the post-processing remained insensitive to the appearance of secondary vortical structures. In the absence of secondary structures, the locations of peak vorticity and minimum tangential velocity typically agreed to within the grid resolution.

As a consequence of the wandering corrections, the resultant instantaneous velocity fields may be, to some extent, discontinuous and therefore nonphysical; the resolution of individual structures from the fields is therefore precluded. However, as discussed above, it is well-accepted that vortex wandering introduces only large-scale, low-amplitude modulations in the vortex trajectory without significantly affecting the vortex dynamics. Because the correction procedure is effectively a high-pass filtering process, its influence will be limited to very low wavenumbers and will therefore not significantly affect the velocity statistics (relative to a vortex not subjected to wandering).

Once the instantaneous velocity fields were corrected for wandering, velocity moments were obtained by spatial averaging along the $z$-axis. Because the vortex and background turbulence were freely decaying, temporal averages could not be defined. These spatiallyaveraged statistics may, however, be directly compared to temporal statistics obtained in a trailing vortex flow by applying Taylor's hypothesis.

\section{Results}

\subsection{Validation}

The numerical method and synthetic turbulence field used for the initial condition were validated by simulating the natural decay of the initial turbulence in the absence of the vortex. The homogeneous, isotropic turbulence field was initialized using (2.3) with $k / 2 U_{\infty}^{2}=6.0 \times 10^{-4}$ (where $k$ is the turbulent kinetic energy and $U_{\infty}$ is the integral velocity scale), in a structured, periodic cube of length $2 \pi$ having 64 evenly-spaced grid points in each direction. The simulation was run at a Reynolds number $R e=L_{\infty} U_{\infty} / \nu=1660$ (where $L_{\infty}$ is the integral length scale), corresponding to a Taylor-microscale Reynolds number $R e_{\lambda}=11.6$. The simulation was run for a total of $\sim 4 \times 10^{4}$ time steps of nondimensional time $t U_{\infty} / L_{\infty}=2.5 \times 10^{-3}$. These initial conditions and nondimensional test parameters were selected in order to match those of Dauzats et al. (2002) obtained using the CERFACS NTMIX code (for details on the implementation, see Hélie 2001), thereby permitting the direct comparison of the present results against this available DNS database.

Figure 2 (a) shows the evolution of the total $k$ with the nondimensional time $t / \tau$ (where 

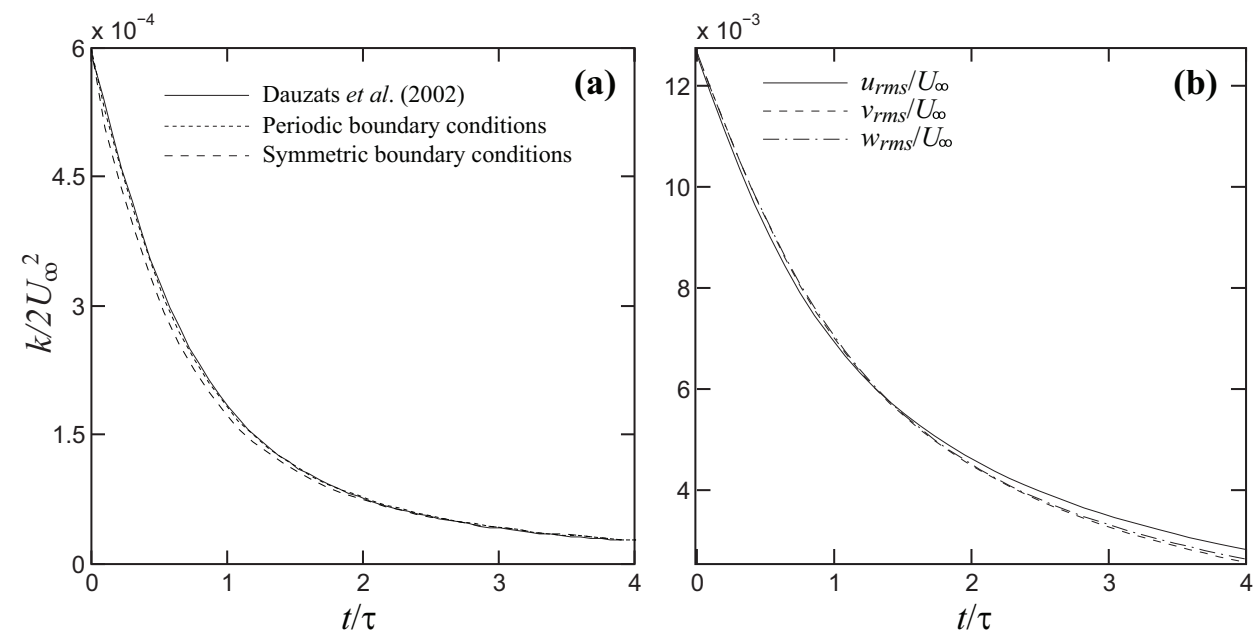

FiguRE 2. Results from simulation of freely decaying synthetic homogeneous isotropic turbulence. (a), Time-evolution of total turbulent kinetic energy; (b), time-evolution of RMS velocity components (within periodic boundary conditions).

$\tau=\Sigma\left(L / u^{\prime 2}\right)$ is the global autocorrelation time scale, $L$ is the local auto-correlation length scale and $u^{\prime 2}$ is the standard deviation of the velocity field at $t=0$ ), along with the available data for comparison, both from simulations having periodic and symmetric boundary conditions. The curves agree very well over the range of at least $t / \tau<4$ (at which time $k / 2 U_{\infty}^{2}$ has decayed to $\sim 4.3 \%$ of its initial value).

The isotropy of the turbulence was also assessed by examining the time-evolution of the individual normalized Cartesian root-mean-square (RMS) velocity components $u_{r m s} / U_{\infty}, v_{r m s} / U_{\infty}$ and $w_{r m s} / U_{\infty}$ taken over the entire domain for the case of the periodic boundary conditions (Figure $2 \mathrm{~b}$ ). The three RMS velocities collapse to within $2 \%$ over the range $t / \tau \lesssim 4$, demonstrating that the isotropy (and, by inference, the homogeneity) of the initial synthetic turbulence field is preserved to at least the second moment of velocity. These results are also indistinguishable from those of Dauzats et al. (2002) (not shown).

\subsection{Time-evolution of a Batchelor vortex}

Direct numerical simulation results were obtained for the case of the velocity fields initialized with (1.1) and (1.2), superimposed upon the random turbulence field having the energy spectrum given by (2.3), using conventional periodic boundary conditions. Figure 3 (a) shows isocontours of $\zeta_{z} r_{c} / V_{0}$ (where $\zeta_{z}=\partial v / \partial x-\partial u / \partial y$ is the cross-flow vorticity), taken at the centre plane of the domain at nondimensional times $t / T=0.19,0.96$ and 2.87 (where $T=2 \pi r_{c} / V_{0} \sim 0.56 \tau$ is the vortex turnover time). Early in the simulation, the peak normalized vorticity closely approximates $1+2 \alpha$ and the vortex appears to be highly axisymmetric for $\eta \lesssim 1$. At $t / T=0.96$, the core remains well-defined and fairly axisymmetric. The vortex has also begun to organize the background turbulence, winding it around the outer core region $(1 \lesssim \eta \lesssim 3)$; this behaviour is consistent with the results presented by Melander \& Hussain (1993). Some distortion of the vortex core is also apparent, as the $\zeta_{z} r_{c} / V_{0} \geq 1$ contour lines approximate ellipses. By $t / T \sim 2.87$, the core has begun to destabilize and lose axisymmetry. Three distinct lobes are evident in the $\zeta_{z} r_{c} / V_{0} \geq 2$ contour lines, consistent with the formation of three co-rotating helical structures within the core. 
To assess the turbulence intensity within the cross-flow plane independently of the location of the vortex centre (upon which the transformation of the Cartesian velocity components into polar coordinates depends strongly), a cross-flow turbulence intensity $u_{z}^{\prime}$ was defined as

$$
u_{z}^{\prime}=\left(\frac{\left\langle u^{2}\right\rangle+\left\langle v^{2}\right\rangle}{\langle U\rangle^{2}+\langle V\rangle^{2}}\right)^{1 / 2},
$$

where $u$ and $v$ are the turbulent defect velocities and $U$ and $V$ are the total velocities in the $x$ and $y$ directions, respectively, and the angled parentheses indicate a spatial average along the $z$-axis. Note that this definition will necessarily result in a singularity at the vortex centre, where the cross-flow velocity magnitude vanishes. Again, the results show an initial random distribution of turbulence (Figure $3 \mathrm{~b}$ ), with fairly low values around the vortex centre relative to the cases at later $t / T$ (the localized concentrations of $u_{z}^{\prime}$ near the vortex centre may be attributed to the singularity in (3.1)), as the near solid-body rotation stabilizes the fluctuations. At $t / T=0.96$, slightly higher levels of cross-flow turbulence are evident in the core region, as secondary structures are wound into the vortex and transport turbulence toward the vortex centre. For large $t / T$, high levels of turbulence are evident at the vortex centre, suggestive of production; however, this is more likely the result of a low-wavenumber modulation stemming from the lobe-like asymmetry of the core seen in Figure 3 (a).

In previous DNS simulations, vortices have been shown to exhibit three distinct stages of development based on the evolution of $k$. Initially, the vortex will undergo a phase of linear growth, during which ambient turbulent structures are organized and concentrated by the mean rotational velocity as the vortex adjusts to the initial and boundary conditions. Once the vortex begins to respond to the influence of the boundary conditions, the vortex transitions to a state of saturation. During this stage, the total turbulent kinetic energy remains relatively constant, but the vortex core undergoes structural changes as it reorganizes and adjusts to the boundary conditions. Finally, the vortex will begin to slowly decay as kinetic energy is lost to viscosity. The vortex will also become increasingly sensitive to the boundary conditions as it grows to the point that the assumption that $r_{c} \ll L_{0}$ (where $L_{0}$ is the integral length scale) weakens.

Figure 4 shows the evolution of the normalized total turbulent kinetic energy $\Sigma k(x, y, z) / 2 V_{0}^{2}$ as a function of time both with and without correction for the effects of vortex wandering. Results from simulations using both symmetric and periodic boundary conditions are shown. The choice of boundary conditions can have a significant impact on $k$; before correcting for wandering, the simulation with symmetric boundary conditions predicted a very linear increase in $k$ from $0 \leq t / T \lesssim 2$, and a gradual transition from $t / T \gtrsim 2$. On the other hand, the periodic boundary conditions resulted in a less linear growth, followed by a clear plateau for $2.5 \lesssim t / T \lesssim 4$. Once corrected for wandering, though, the results collapse independent of the boundary conditions for $t / T \lesssim 2.5$, suggesting that the boundary conditions have not yet exerted a major influence upon the vortex development (or that the influence of the boundary conditions is effectively removed by correcting for wandering). For $t / T>2.5$, the difference between the corrected results increases, but remains small relative to the uncorrected cases; also, both curves exhibit a small plateau followed by a clear decrease in $k / 2 V_{0}^{2}$, consistent with the transition to saturation.

These results provide compelling evidence that the vortex wandering occurs as a consequence of the boundary conditions. Also, including the effects of this large-scale, inactive wandering in estimates of turbulence intensity not only result in a significant, boundary 

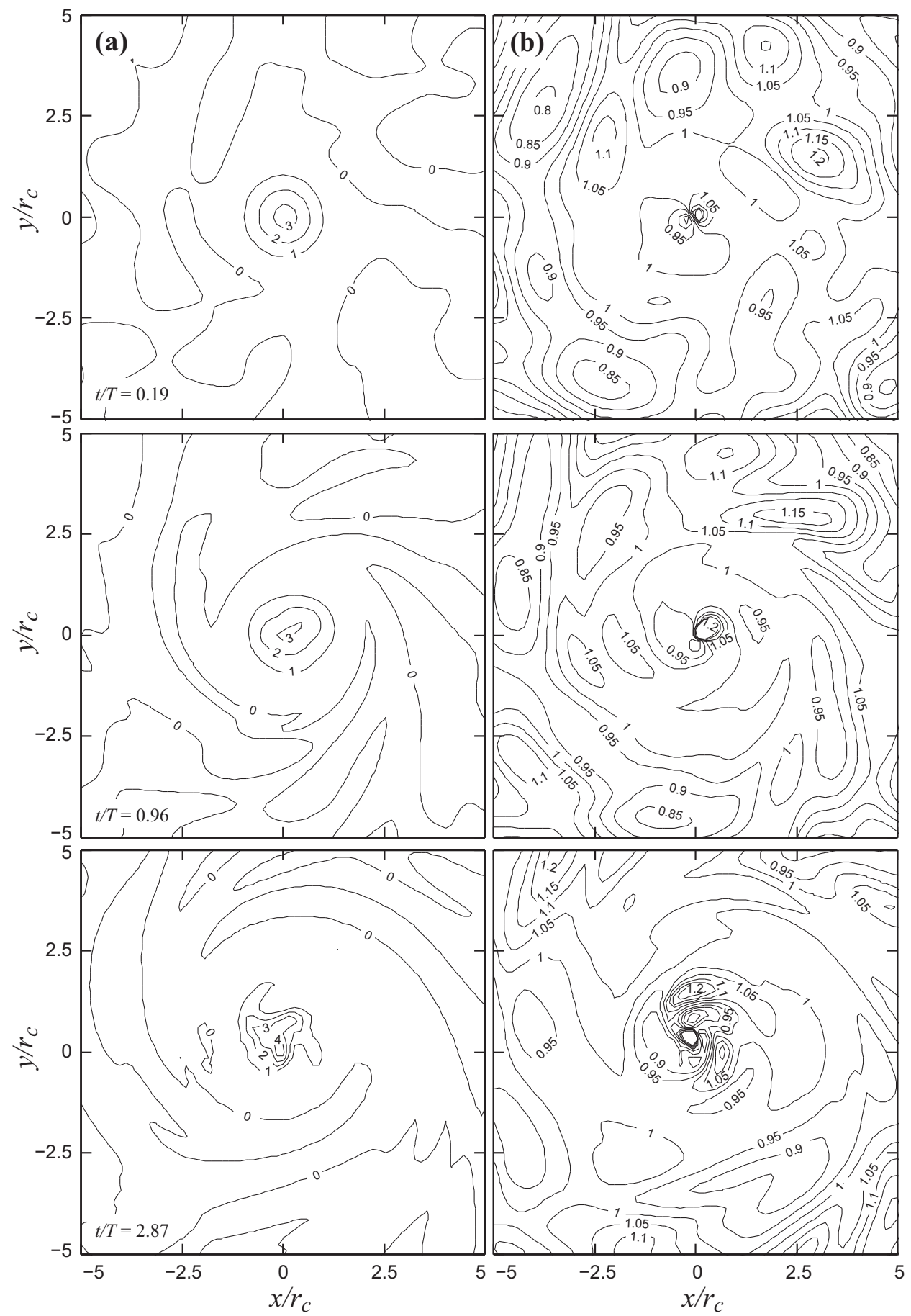

Figure 3. Contours of normalized (a) cross-flow vorticity, and (b) cross-flow turbulence intensity at $t / T=0.19,0.96$ and 2.87 . 


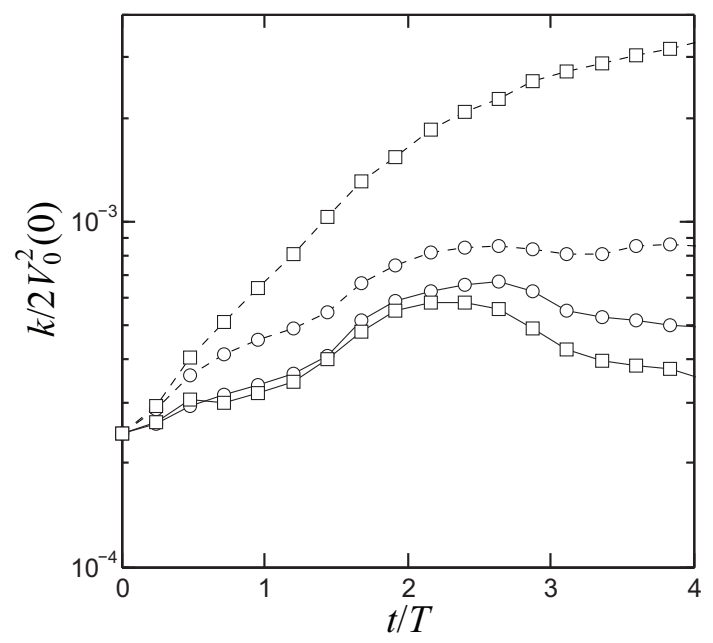

Figure 4. Evolution of normalized total turbulent kinetic energy. ०, Periodic boundary conditions; $\square$, symmetric boundary conditions; - - -, uncorrected; $\stackrel{-}{-}$, corrected. Symbols show every 1000 th time step.

condition-specific (and therefore arguably artificial) increase in $k$, but the characteristic stages of vortex evolution may be entirely masked.

Since the effect of vortex wandering is primarily to distort the vortex trajectory in both space and time, the trajectories are of particular interest and warrant some additional attention. Figure (5) shows the vortex trajectories at $t / T=0.72,1.44$ and 2.40 for both the cases of periodic and symmetric boundary conditions. Early in the vortex development, the trajectory is characterized by fairly small disturbances concentrated within limited regions of $z / r_{c}$. As the vortex ages, larger-amplitude, wave-like deviations having amplitudes of $\sim 0.2 r_{c}$ and wavelengths of $\sim 15 r_{c}$ begin to form. A second mode of deviation, in the form of short-wavelength, rapid displacements also emerges. These discrete 'jumps' occur as the criteria used to identify the vortex centre select between multiple candidate points within an increasingly asymmetric vortex core. It is interesting to note that the larger-wavelength modulations appear to be fairly independent of the boundary conditions, while the 'jumps' are more frequent under symmetric boundary conditions. These results suggest that the clear boundary condition-dependence in the uncorrected curves presented in Figure 4 occurs as a consequence of fairly subtle structural differences in the core region of the vortex.

The effect of the boundary conditions upon the time evolution of the vortex trajectories is more clearly demonstrated in Figure 6 (a), which shows the peak normalized vortex excursion $\Delta r(z ; t) / r_{c}$ (defined as the maximum displacement of the vortex centre from its initial condition), as well as the standard deviation of normalized vortex excursion $\sigma / r_{c}$. The peak excursions are significantly smaller and increase more gradually with time for the case of the periodic boundary conditions. For $t / T \lesssim 2.5$, excursions by as much as $0.3 r_{c}$ and $0.5 r_{c}$ are apparent for the cases of the periodic and symmetric boundary conditions, respectively. Through most of the range $0.5 \lesssim t / T \lesssim 2.5, \sigma / r_{c} \sim 0.07$ for both boundary conditions.

For the case of an axisymmetric Batchelor vortex undergoing an isotropic and purely Gaussian wandering about the origin, Devenport et al. (1996) showed that the wandering tends to act as a convolution filter with a Gaussian kernel, so that the maximum tangential velocity $\overline{V_{0}}$ of the spatially-averaged vortex varies from the true peak tangential 


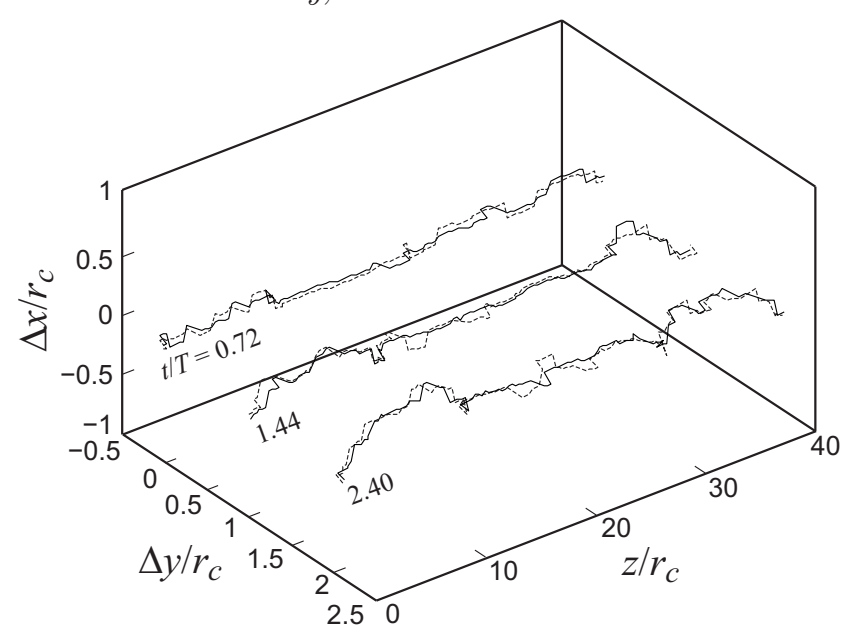

FIGURE 5. Trajectory of vortex centre normalized against initial core radius, at $t / T=0.72$, 1.44 and 2.40. - Periodic boundary conditions; - - -, symmetric boundary conditions. Mean paths have been offset in $y$ for clarity.
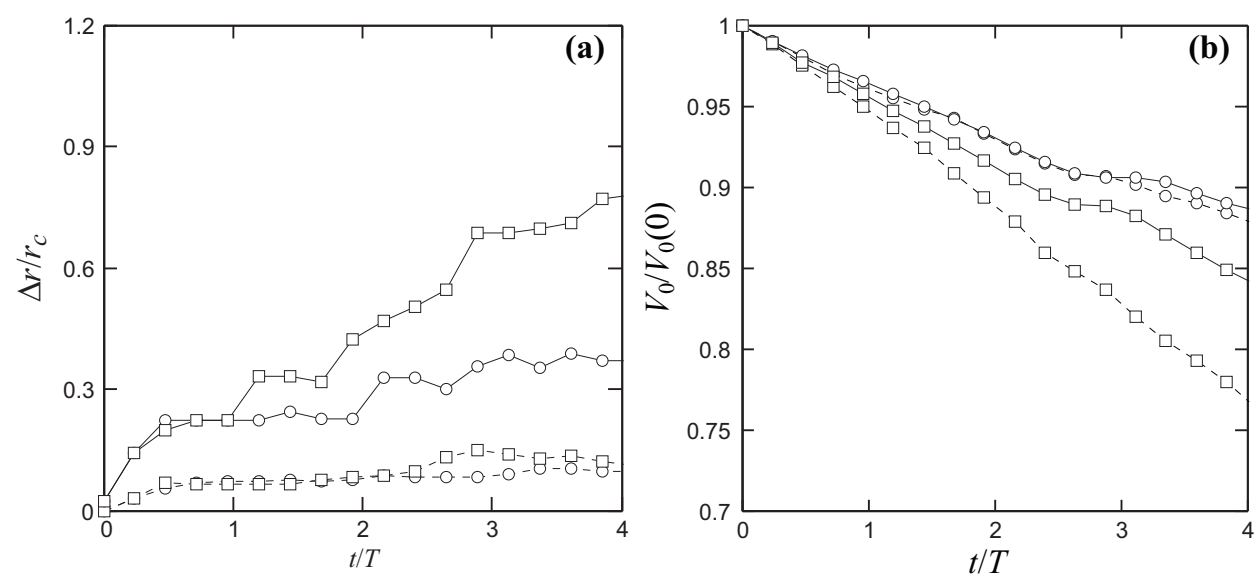

Figure 6. (a) Evolution of wandering amplitude with time. o, Periodic boundary conditions; $\square$, symmetric boundary conditions;,$- \Delta r / r_{c}$; - - -, $\sigma / r_{c}$. (b) Evolution of $V_{0}$ with time. $\circ$, Periodic boundary conditions; $\square$, symmetric boundary conditions; - - -, uncorrected; - corrected. Symbols show every 1000th time step.

velocity as

$$
\frac{\overline{V_{0}}}{V_{0}}=\left(1-2 \alpha \frac{\sigma^{2}}{r_{c}^{2}}\right)^{1 / 2} .
$$

For $\sigma / r_{c} \sim 0.07,(3.2)$ predicts that the convolution will result in a reduction of peak velocity of less than 1\%. Figure 6 (b) shows the evolution of peak tangential velocity with time. For the case of the periodic boundary conditions, the wandering correction has a negligible effect upon $V_{0}$ for $t / T \lesssim 4$, consistent with the prediction of (3.2). For the case of the symmetric boundary conditions, the difference between the corrected and uncorrected peak tangential velocities is as large as $10 \%$, and an order of magnitude larger than the difference predicted by (3.2). Since (3.2) necessarily must be satisfied if the wandering is isotropic and Gaussian, and since statistical anisotropy is unlikely 

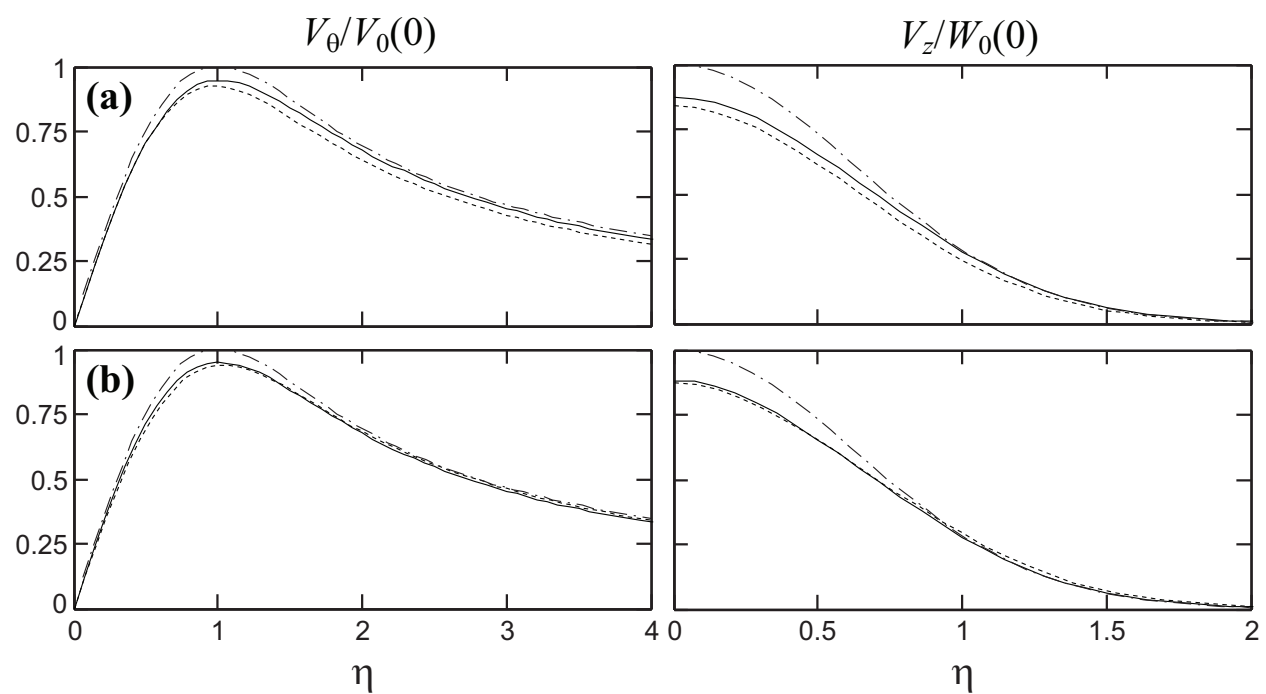

FiguRE 7. Circumferentially averaged radial profiles of tangential and axial velocity at $t / T \sim$ 1.44. (a), uncorrected; (b) corrected. — , Periodic boundary conditions; - - -, symmetric boundary conditions; - - - , (1.1) or (1.2).

given the symmetry of the problem, the failure of (3.2) suggests that vortex wandering within symmetric boundary conditions is non-Gaussian. It is also important to note that, once corrected for the effects of wandering, the peak tangential velocities in this case are increased by $\sim 5 \%$.

\subsection{Mean velocity components}

In order to examine the effect of boundary conditions upon the spatially-averaged velocity statistics, the circumferentially-averaged profiles of the velocity components were evaluated at $t / T \sim 1.44$, when the vortex had sufficient time to develop from the initial condition, but is still at a stage where it would be expected to remain only minimally affected by the presence of the boundaries. Figure 7 shows the tangential and axial velocity profiles (normalized against the velocity magnitude from the initial condition), as well as the Batchelor profile from the initial condition for comparison. The peak velocities have decayed, as $r_{c}$ increased with vortex age. The periodic boundary conditions result in slightly higher uncorrected magnitudes, though the first-order velocity statistics show only a small boundary-condition sensitivity $(\sim 3 \%)$. The profiles collapse throughout the domain once corrected.

The relative insensitivity of the mean velocity profiles to the random wandering is, to some extent, expected; it has already been shown that the form of a Batchelor vortex is preserved through a Gaussian convolution (Devenport et al. 1996; Birch 2012). If the wandering in the case of the symmetric boundary conditions is non-Gaussian, this is not causing a significant variation in the expected velocity profile.

\subsection{Reynolds stresses}

The second-order velocity statistics exhibit a much stronger sensitivity to the boundary conditions and response to vortex wandering. Figure 8 shows the normalized, circumferentially-averaged polar Reynolds stresses $\left\langle v_{\theta}^{2}\right\rangle / V_{0}^{2},\left\langle v_{r}^{2}\right\rangle / V_{0}^{2}$ and $\left\langle v_{z}^{2}\right\rangle / V_{0}^{2}$ (where $V_{0}$ here is taken from the initial condition) for both boundary conditions, with and without correction for wandering. In all cases, the uncorrected stresses exhibit much larger 

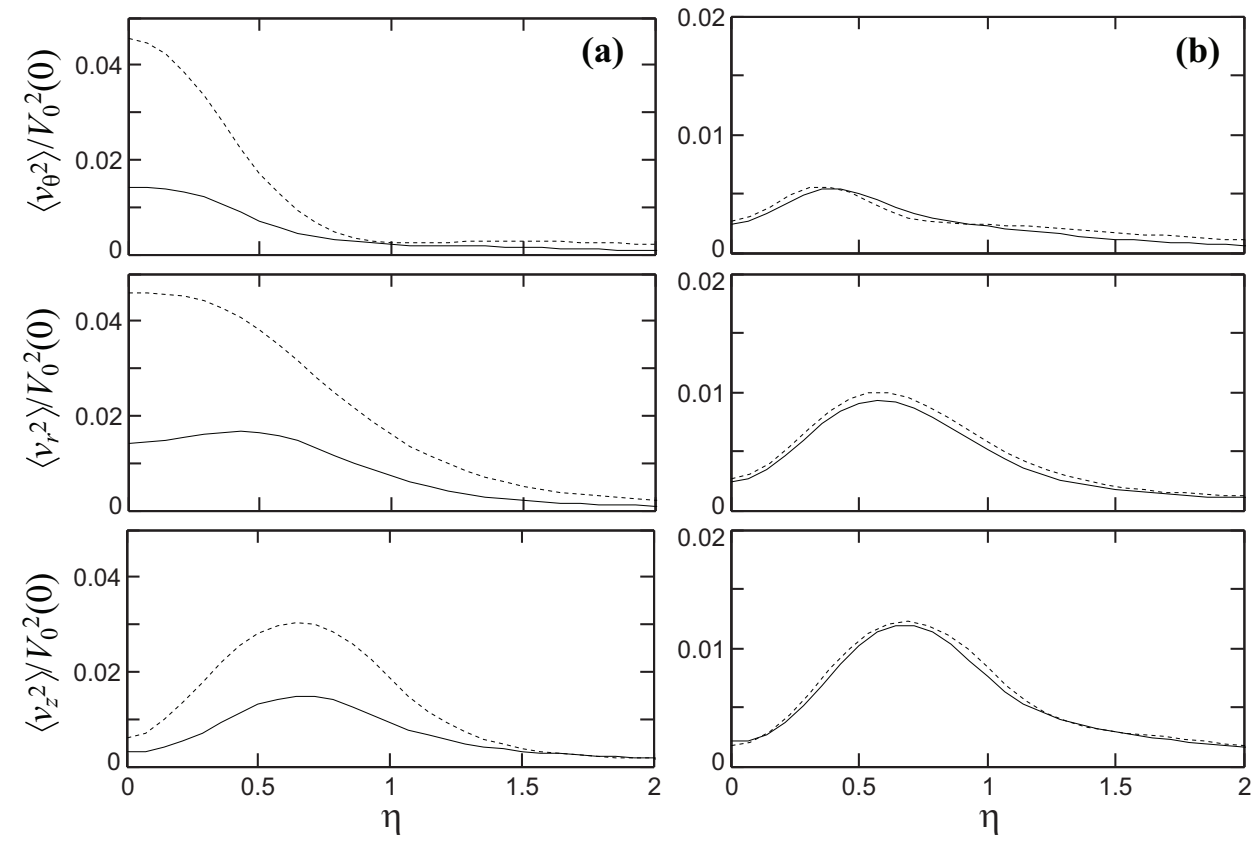

FIGURE 8. Circumferentially averaged radial profiles of Reynolds stresses at $t / T \sim 1.44$. (a), uncorrected; (b) corrected. ——, Periodic boundary conditions; - - -, symmetric boundary conditions.

peak magnitudes, demonstrating that the inactive wandering is contributing significantly to the measured stresses. Furthermore, the uncorrected profiles are strongly boundarycondition dependent but collapse when corrected, providing additional evidence to support the concept that vortex wandering is driven primarily by boundary conditions.

The radial distributions of Reynolds stress are also strongly affected by wandering. Uncorrected profiles of $\left\langle v_{\theta}^{2}\right\rangle$ and $\left\langle v_{r}^{2}\right\rangle$ both exhibit local maxima at or near the vortex centre, and are approximately Gaussian in form. However, this distribution is necessarily an artifact of wandering; once corrected, both the tangential and radial Reynolds stresses drop to negligible levels at the origin, with the peak shifted to $0.4 \lesssim \eta \lesssim 0.6$. This corresponds qualitatively to the form of uncorrected $\left\langle v_{z}^{2}\right\rangle$; since vortex wandering is essentially a cross-flow phenomenon, the effect of boundary conditions and wandering upon the form of $\left\langle v_{z}^{2}\right\rangle$ is expected to be marginal.

The importance of the boundary conditions in determining the vortex behaviour is further illustrated in Figure 9, which shows the radial distributions of $\left\langle v_{\theta}^{2}\right\rangle / V_{0}^{2}$ at a time $t / T \sim 2.4$; results at this $t / T$ are directly comparable to the DNS data presented by Qin (1998). As expected, the available DNS data agreed reasonably well with the present results for the case of the periodic boundary conditions; the magnitudes were similar, with very low gradients near the vortex centre and through into the core region. The solutions begin to differ for $\eta \gtrsim 0.5$, though this is likely due to differences in the both the domain size and the initial and boundary conditions: Qin (1998) used only $15 \times 15$ points in the cross-flow plane, and implemented some inviscid modelling near the boundaries as well (see also Duraisamy \& Lele 2008). Since solutions express boundary condition sensitivity at this $t / T$, some disagreement at larger $\eta$ between models is expected.

Figure 9 also includes (uncorrected) experimental data from Bailey \& Tavoularis (2008), from high-bandwidth, single-point measurements of a vortex formed in varying levels of grid-generated background turbulence. These measurements were collected 


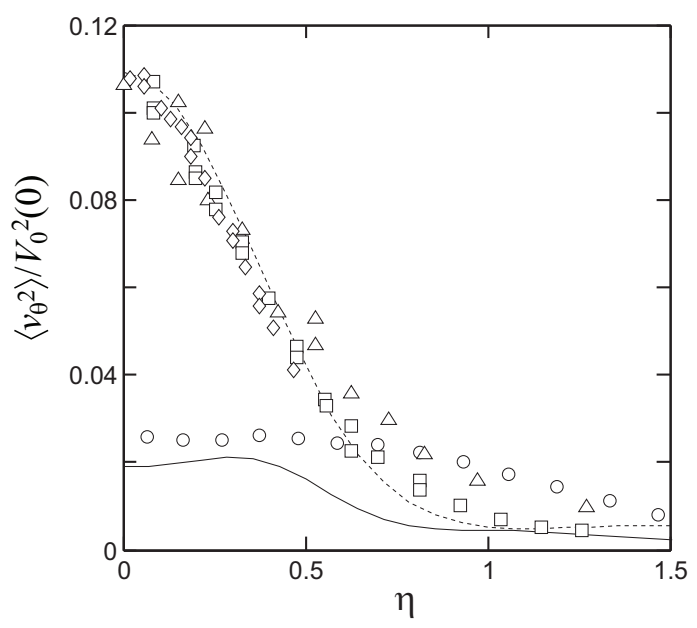

Figure 9. Comparison of $\left\langle v_{\theta}^{2}\right\rangle / V_{0}^{2}$ at $t / T \sim 2.4$. o, Qin (1998); other symbols, rescaled cases taken from Bailey \& Tavoularis (2008); —_, periodic boundary conditions; - - -, symmetric boundary conditions.

using multi-sensor hot-wire probes, in a facility measuring approximately $65 r_{c} \times 100 r_{c}$ in the cross-flow plane. Because the background turbulence intensity and vortex 'age' could not be matched, a direct, quantitative comparison to the present results cannot be made; however, the form of these distributions may be considered by rescaling the results. Although the rescaling is arbitrary, three different cases from Bailey \& Tavoularis (2008) are shown, having upstream turbulence intensities $k / 2 V_{0}^{2} \sim 5.0 \times 10^{-3}, 2.0 \times 10^{-3}$ and $1.9 \times 10^{-5}$. Once appropriately rescaled, these cases all collapse; this demonstrates that the form of the solution is independent of the vortex state and magnitude of the background turbulence. Furthermore, the rescaled data agree well with the uncorrected results of the present symmetric boundary condition case, and, independent of the radial scaling, no plateau is apparent in the core region of the experimental data. Aside from providing some validation for the present simulations, these results also provide a good example of the poor agreement between experiment and simulation which can become evident in the higher-order velocity statistics, and suggest that this is, again, due to the effect of boundary conditions.

Figure 10 shows the normalized polar Reynolds shear stresses $\left\langle v_{\theta} v_{r}\right\rangle / V_{0}^{2},\left\langle v_{\theta} v_{z}\right\rangle / V_{0}^{2}$ and $\left\langle v_{r} v_{z}\right\rangle / V_{0}^{2}$. Both $\left\langle v_{\theta} v_{r}\right\rangle$ and $\left\langle v_{r} v_{z}\right\rangle$ are an order of magnitude smaller than the other Reynolds stresses. The forms of the distributions and the amplitudes are not significantly affected by correction relative to the other Reynolds stress components, suggesting that the radial velocity fluctuations are at most only weakly correlated to the axial and tangential components. It is interesting to note, however, that these Reynolds stresses nearly vanish within symmetric boundary conditions, but show small local extrema at $\eta \lesssim 1$ within periodic boundary conditions.

The axial-tangential Reynolds shear stress, on the other hand, demonstrates boundarycondition sensitivity, but again collapses when corrected. A negative peak is apparent at $\eta \sim 0.5$ in both the uncorrected and the corrected profiles, though the wandering significantly increases the apparent amplitude.

Figure 11 shows the evolution of the peak circumferentially-averaged Reynolds stresses with time both with and without the correction for wandering. For the case of the symmetric boundary conditions, the wandering clearly dominates in the peak Reynolds stresses, as the cross-flow components $\left\langle v_{\theta}^{2}\right\rangle$ and $\left\langle v_{r}^{2}\right\rangle$ collapse, and achieve amplitudes an 

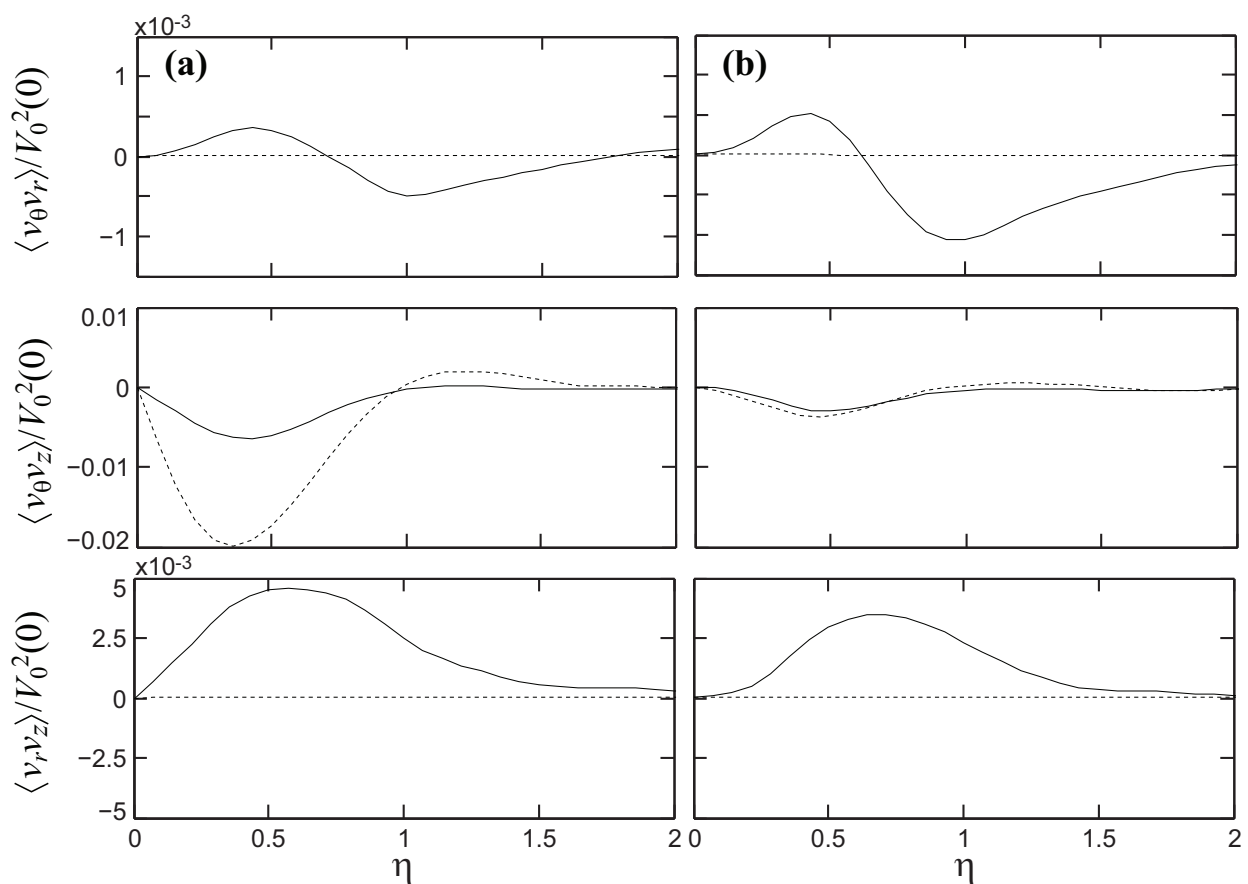

FIGURE 10. Circumferentially averaged radial profiles of Reynolds stresses at $t / T \sim 1.44$. (a), uncorrected; (b) corrected. ——, Periodic boundary conditions; - - -, symmetric boundary conditions.

order of magnitude larger than when corrected. Within the periodic boundary conditions, all three Reynolds stress maxima collapse for $t / T \lesssim 1.5$. For $t / T \gtrsim 1.5$, the radial component increases in magnitude, and collapses again with the tangential component for $t / T \gtrsim 3$.

Once corrected for wandering, the peak Reynolds stresses become largely boundary condition-independent. A clear maximum was apparent at around $2 \lesssim t / T \lesssim 3$ for all three orthogonal components, which is consistent with the broad maximum in $k$ (see Figure 4). For $t / T \gtrsim 3$, the strong differences between the Reynolds stresses diminish as the turbulence decays.

More revealing, perhaps, is the time-map of the radial distribution of the corrected Reynolds stresses. Figure 12 shows the evolution of the circumferentially-averaged Reynolds stresses in both space and time, so that the curves shown in Figure 10 represent horizontal sections through the iso-surfaces plotted (at $t / T=1.44$ ), while the curves in Figure 11 represent vertical sections along a line of maximum local stress.

Figure 12 (a) shows contours of $\left\langle v_{\theta}^{2}\right\rangle / V_{0}^{2}$, as a function of both radius and time. The peak begins to manifest at $t / T \sim 1$, but grows rapidly with time. By $t / T \sim 2$, a second peak forms at $\eta \sim 1$, and persists through to $t / T \sim 3$. Similar peaks were not observed in the distributions of the $\left\langle v_{r}^{2}\right\rangle / V_{0}^{2}$ or $\left\langle v_{z}^{2}\right\rangle / V_{0}^{2}$ (Figures $12 \mathrm{~b}-\mathrm{c}$ ), though a distinct increase in skewness toward larger $\eta$ is apparent in these distributions, especially for $t / T \gtrsim 2$. The magnitudes of $\left\langle v_{\theta} v_{r}\right\rangle$ (Figure $12 \mathrm{~d}$ ) are small compared to the other stresses (as demonstrated in Figure 10). While the amplitudes of $\left\langle v_{\theta} v_{z}\right\rangle$ and $\left\langle v_{r} v_{z}\right\rangle$ are still fairly small, strong local maxima are nevertheless apparent in these distributions at $\eta \sim 0.5$ and 1, respectively (Figure 12 e-f). Note that, of all the Reynolds stresses, $\left\langle v_{r} v_{z}\right\rangle$ exhibits the most significant skewness, with the peak drifting toward larger $\eta$ with increasing time. 

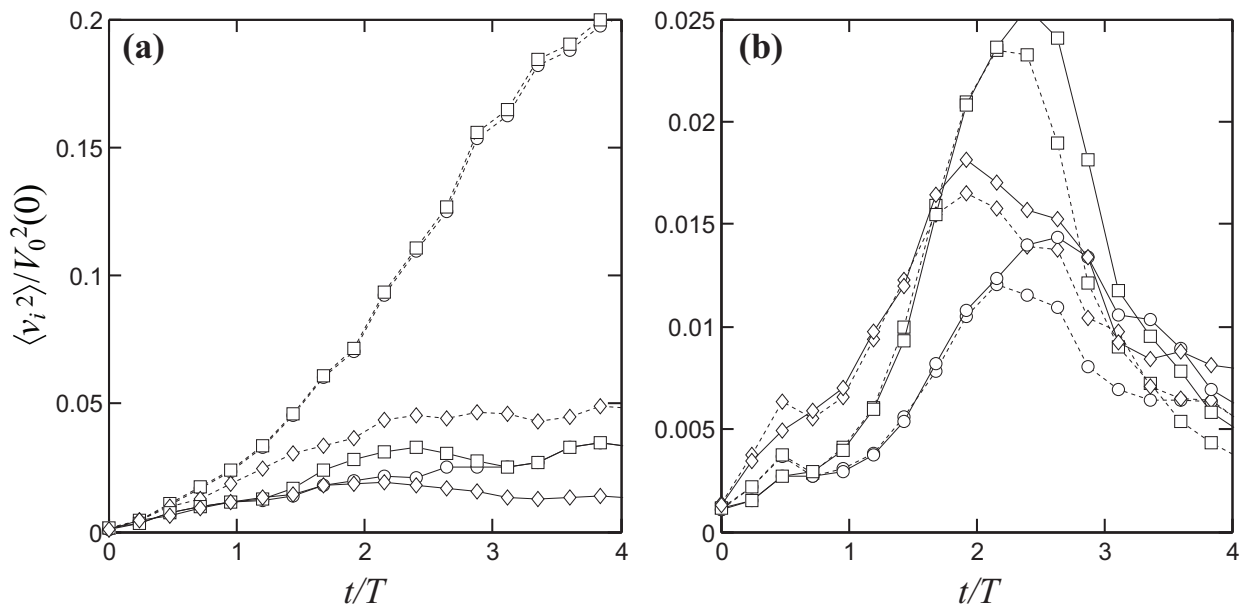

FiguRE 11. Evolution of peak circumferentially-averaged Reynolds stresses (a) without correction and (b) with correction. o, $\left\langle v_{\theta}^{2}\right\rangle / V_{0}^{2} ; \square,\left\langle v_{r}^{2}\right\rangle / V_{0}^{2} ; \diamond,\left\langle v_{z}^{2}\right\rangle / V_{0}^{2}$. $\longrightarrow$, Periodic boundary conditions; - - -, symmetric boundary conditions. Symbols show every 1000th time step.

\section{Discussion}

The effect of vortex wandering upon the mean velocities in a turbulent vortex is not significant. Owing to the robustness of (1.1) and (1.2) in describing vortices and their invariance through the convolution, it is expected that the isotropic, low-wavenumber and low-amplitude Gaussian modulations characteristic of wandering will have a marginal effect. However, the absence of a universal scaling for the higher-order velocity statistics within vortex flows suggests that these quantities may be more sensitive to wandering. Because nearly all of the second-order statistics obtained showed strong boundary condition-dependence that was eliminated by correcting for wandering, a compelling argument can be made that the wandering occurs as a direct consequence of the combined action of boundary conditions and background turbulence. The symmetric boundary conditions (which most closely model physical boundaries) produced wandering amplitudes significantly larger than the periodic boundary conditions commonly used in spectraldomain simulations, and had a pronounced effect on the uncorrected statistics. The good agreement between the (uncorrected) velocity moments from the symmetric boundary condition case and rescaled, uncorrected experimental data further reinforce this point. The direct comparison of DNS results obtained using conventional spectral methods and experimental data is therefore not valid, at least for velocity statistics above the first moment.

As a consequence of the 'inactive' modulation introduced by wandering, the cross-flow Reynolds stresses developed a strong peak at the centre of the vortex. If the wandering is considered as part of the vortex evolution, this might be interpreted as a strongly turbulent core. However, once the velocities have been corrected for wandering, very low levels of turbulence are recovered near the centre of the vortex. Of more interest, perhaps, is the strong peak in Reynolds stresses which emerges away from the vortex centre but within the core region. This provides evidence of the presence of energetic secondary structures within the core, which is normally taken to have a strongly stabilizing effect on turbulence. Weaker, distributed peaks in the Reynolds stresses are apparent at around $\eta \sim 1$ for $t \gtrsim 2$. These, with the strong cross-correlations between the different velocity components at different radii, are consistent with the 'hairpin' structures identified by Duraisamy \& Lele (2008), while the very poor correlation between the radial 


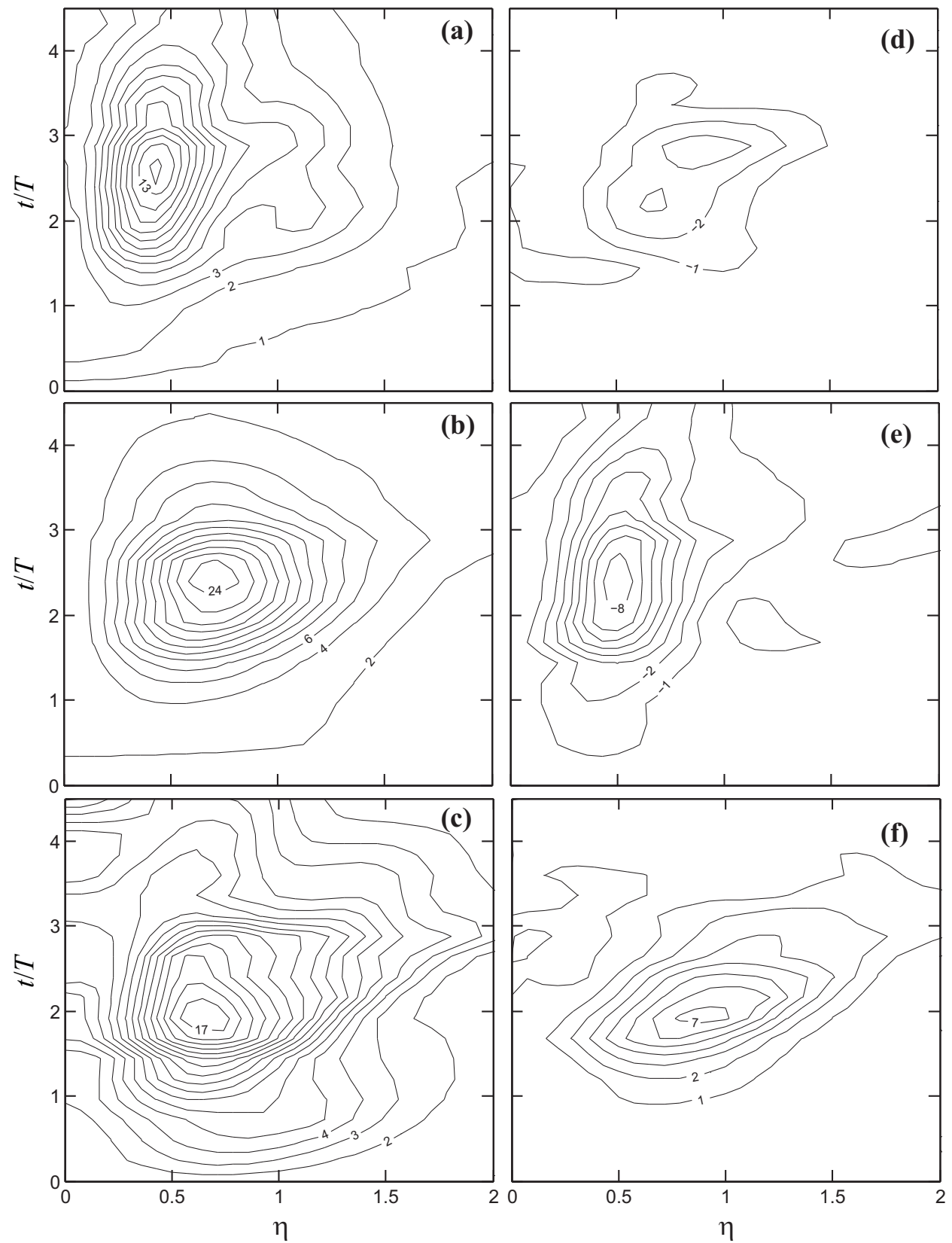

Figure 12. Contours of (a) $\left\langle v_{\theta}^{2}\right\rangle / V_{0}^{2} \times 10^{3}$, (b) $\left\langle v_{r}^{2}\right\rangle / V_{0}^{2} \times 10^{3}$, (c) $\left\langle v_{z}^{2}\right\rangle / V_{0}^{2} \times 10^{3}$, (d) $\left\langle v_{\theta} v_{r}\right\rangle / V_{0}^{2} \times 10^{3}$, (e) $\left\langle v_{\theta} v_{z}\right\rangle / V_{0}^{2} \times 10^{3}$ and (f) $\left\langle v_{r} v_{z}\right\rangle / V_{0}^{2} \times 10^{3}$, showing evolution of profiles in both space and time. Data from the periodic boundary condition case, corrected for wandering.

and tangential velocity components indicate that the dominant secondary structures in the core flow are not aligned with the primary vortex. Taken together with the overall increase in turbulent kinetic energy for $t / T \lesssim 2.5$, this provides further evidence that the main mechanism of turbulent production during this stage of vortex development is the formation and organization of these secondary structures within the core. 
It is also significant to note here that, once corrected for vortex wandering, the radial profiles of Reynolds stresses (scaled against the instantaneous core radius) demonstrate some time-dependence; peak values slowly drifted towards larger $\eta$ and some bi-modality emerged with increasing time. This suggests that the strong self-similarity of turbulent vortices demonstrated by Birch (2012) may not necessarily extend to the higher-order velocity statistics.

\section{Conclusion}

A direct numerical simulation of a turbulent vortex evolving in naturally decaying background turbulence has been carried out using both periodic and symmetric boundary conditions. The results were shown to be strongly dependent on the boundary conditions, but collapsed once the results were corrected for the random vortex 'wandering' using a technique common in the analysis of experimental vortex data. Vortex wandering was therefore shown to be strongly influenced by the boundary conditions, and that the modelling of a free vortex by a 'vortex in a box' may not be valid, even if the effect of the walls upon the vortex appears to be small at the leading order.

Because vortex wandering is a low-wavenumber Gaussian modulation, it is expected to play only a passive role in the development of turbulent vortices. Once the effect of vortex wandering was removed from the velocity statistics, the Reynolds stresses began to more accurately reflect the true structure of the vortex. Strong local maxima were apparent within the core region in all of the circumferentially-averaged Reynolds stress distributions, with the notable exception of the radial-tangential component. These Reynolds stress distributions were consistent with the presence of organized, secondary structures within the core region which were primarily responsible for turbulent production.

\section{Acknowledgments}

This work was supported in part by the U.K. Engineering and Physical Sciences Research Council under grant number EP/H030360/1.

\section{REFERENCES}

Bailey, S. C. C. \& Tavoularis, S. 2008 Measurements of the velocity field of a wing-tip vortex, wandering in grid turbulence. J. Fluid Mech. 601, 281-315.

BAtchelor, G. K. 1964 Axial flow in trailing line vortices. J. Fluid Mech. 20, 645-658.

Beninfti, M. \& Marshall, J. 2005 An experimental study of the effect of free-stream turbulence on a trailing vortex. Exp. Fluids 38, 244-257.

Birch, D. M. 2012 Self-similarity of trailing vortices. Phys. Fluids 24 (2), 025105.

Birch, D. M. \& LEE, T. 2005 Investigation of the near-field tip vortex behind an oscillating wing. J. Fluid Mech. 544, 201-241.

Birch, D. M., Lee, T., Mokhtarian, F. \& Kafyeke, F. 2004 Structure and induced drag of a tip vortex. J. Aircraft 41 (5), 1138-1145.

Birch, D. M. \& Martin, N. 2013 Tracer particle momentum effects in vortex flows. J. Fluid Mech. 723, 665-691.

Bradshaw, P. 1967 Inactive motion and pressure fluctuations in turbulent boundary layers. $J$. Fluid Mech. 30, 241-258.

Dauzats, S., Helie, J., Bedat, B. \& Poinsot, T. 2002 Homogenous isotropic turbulence. DNS Database available from http://www.cerfacs.fr/ ntmix/qpf.html .

Devenport, W. J., Rife, M. C., Liapis, S. I. \& Follin, G. J. 1996 The structure and development of a wing-tip vortex. J. Fluid Mech. 312, 67-106. 
Duraisamy, K. \& Lele, S. K. 2008 Evolution of isolated turbulent trailing vortices. Phys. Fluids 20, 035102.

Froehlich, J., Garcia-Villalba, M. \& Rodi, W. 2008 Scalar mixing and large-scale coherent structures in a turbulent swirling jet. Flow Turb. Comb. 80 (1), 47-59.

Giuni, M. 2013 Formation and early development of wingtip vortices. PhD thesis, University of Glasgow.

Goto, S. 2008 A physical mechanism of the energy cascade in homogeneous isotropic turbulence. J. Fluid Mech. 605, 355-366.

HÉLIE, J. 2001 Numerical simulation and modelling of premixed flame propagation in a richnessstratified medium (in French). PhD thesis, Institut National Polytechnique de Toulouse.

Iungo, G. V., Skinner, P. \& Buresti, G. 2009 Correction of wandering smoothing effects on static measurements of a wing-tip vortex. Exp. Fluids 46, 435-452.

Kroo, I. 2001 Drag due to lift: concepts for prediction and reduction. Ann. Rev. Fluid Mech. 33, 587-617.

Kurihara, Y., Bender, M. A. \& Ross, R. J. 1993 An initialization scheme of hurricane models by vortex specification. Monthly Weather Rev. 121 (7), 2030-2045.

Laizet, S. \& Lamballais, E. 2009 High-order compact schemes for incompressible flows: a simple and efficient method with quasi-spectral accuracy. J. Comput. Phys. 228 (16), 59896015.

Lele, S. K. 1992 Compact finite difference schemes with spectral-like resolution. J. Comput. Phy. 103 (1), 16-42.

McCormick, B., Tangler, J. \& Sherrieb, H. 1968 Structure of trailing vortices. J. Aircraft 5 (3), 260-267.

Melander, M. \& Hussain, F. 1993 Coupling between a coherent structure and fine-scale turbulence. Phys. Rev. E 48 (4), 2669-2688.

Pradeep, D. S. \& Hussain, F. 2004 Effects of boundary condition in numerical simulations of vortex dynamics. J. Fluid Mech. 516, 115-124.

QIN, J. H. 1998 Numerical simulations of a turbulent axial vortex. PhD thesis, Purdue University.

RAmaprian, B. R. \& Zheng, Y. 1998 Near field of the tip vortex behind an oscillating rectangular wing. AIAA J. 36, 1263-1269.

Spalart, P. 1998 Airplane trailing vortices. Ann. Rev. Fluid Mech. 30, 107-138.

Townsend, A. A. 1961 Equilibrium layers and wall turbulence. J. Fluid Mech. 11, 97-120.

van Jahrsveld, J. P. J., Holten, A. P. C., Elsenaar, A., Trieling, R. R. \& van Heijst, G. J. F 2011 An experimental study of the effect of external turbulence on the decay of a single vortex and a vortex pair. J. Fluid Mech. 670, 214-239.

Wang, Z. J. 2005 Dissecting insect flight. Ann. Rev. Fluid Mech. 37, 183-210.

Zhang, W. \& SARKAR, P. P. 2012 Near-ground tornado-like vortex structure resolved by particle image velocimetry. Exp. Fluids 52, 479-493. 